\title{
Passage of low-pathogenic avian influenza (LPAI) viruses mediates rapid genetic adaptation of a wild-bird isolate in poultry
}

\author{
Daniel Dlugolenski • Les Jones • Geraldine Saavedra • \\ S. Mark Tompkins • Ralph A. Tripp • \\ Egbert Mundt
}

Received: 26 August 2010/ Accepted: 10 December 2010/Published online: 1 January 2011

(C) Springer-Verlag 2010

\begin{abstract}
For a better understanding of evolution of influenza viruses, a chicken-origin and wild-bird-origin lowpathogenic avian influenza virus (LPAI) was serially passaged in chickens. Sequences of the hemagglutinin (HA) and neuraminidase (NA) genes at each passage level were compared to those of the parental virus. Multiple mutations occurring early during passage were detected, but these were maintained during passages. Interestingly, a number of the observed mutations already existed in the parental virus, as indicated by the presence of single-nucleotide polymorphisms. The greatest numbers of mutations occurred during passage of wild-bird-origin LPAI, where a 20-amino-acid deletion in the NA gene that was observed during the first passage was maintained during subsequent passages. Subsequent experiments showed that this NA deletion was already present as a minority population in the parental virus. These results showed that a selection process favoring a viral subpopulation had occurred.
\end{abstract}

\section{Introduction}

Avian influenza virus (AIV) is a member of the genus Influenzavirus A, family Orthomyxoviridae, which comprises a

\footnotetext{
D. Dlugolenski · E. Mundt $(\square)$

Department of Population Health,

Poultry Diagnostic and Research Center,

953 College Station Road, Athens, GA, USA

e-mail: emundt@uga.edu

D. Dlugolenski · L. Jones · G. Saavedra ·

S. M. Tompkins · R. A. Tripp

Department of Infectious Diseases, College of Veterinary

Medicine, University of Georgia, Athens,

GA 30602, USA
}

large group of enveloped viruses. The virus contains eight segments of negative-sense single-stranded RNA that encode 11 proteins. Most of the viral proteins are incorporated into the virus particle, except the nonstructural protein NS2 (or nuclear export protein, NEP), which is encoded by the smallest viral RNA segment [18, 19, 28, 32]. Influenza viruses are classified according to their hemagglutinin (HA) and neuraminidase (NA) subtypes. Currently, 16 HA (H 1-16) and nine NA subtypes (N 1-9) of AIV have been identified [9, 17].

Wild birds are the natural reservoir for AIV, but infection of most wild birds is asymptomatic, allowing the virus to circulate in the environment without being noticed [36, 40]. Most AIV strains belong to the low-pathogenic avian influenza (LPAI) type, but some LPAI, in particular those belonging to the HA5 (H5) and HA7 (H7) subtypes, can become highly pathogenic AIV (HPAI). HPAI strains are thought to arise in part due to introduction of a stretch of multiple basic amino acids at the proteolytic cleavage site (polybasic cleavage site) between the HA1 and HA2 subunits of the HA protein, which facilitates not only cleavage by trypsin-like proteases but also cleavage by intracellular proteases such as furin-like proteases, enabling systemic spread of the virus and increasing its virulence $[2,11,14,16$, $22,30]$. It has been reported that the introduction of a polybasic cleavage site in an $\mathrm{H} 3$ or $\mathrm{H} 5$ subtype strain of AIV does not necessarily confer high pathogenicity, suggesting that other factors contribute to the HPAI phenotype [14, 37].

It is recognized that RNA virus populations such as influenza virus consist of complex distributions of mutant genomes in a population structure known as quasispecies [7] and that subpopulations of virulent or better host-adapted viruses may sometimes be selected [8, 13]. Mounting evidence suggests that this occurs in the case of HPAI, which may be present in circulating virus populations for several months before emerging as the major 
virus population $[3,4]$. Similarly, mutations occurring in a parental strain can give rise to the emergence of a virus that is better adapted to the host and has a different phenotype. One recent example is the adaptation of LPAI from wild birds to chickens, as characterized by a truncation of the NA stalk region [21]. The deletion in the NA stalk region during wild-bird-to-poultry adaptation appears to be a hallmark of adaptation in chickens, as this feature was observed in all viruses examined from a H7N1 outbreak in poultry in Italy, where isolates contained an NA stalk deletion, which was not present in the NA genes of the wild-bird virus isolates [1]. In addition, a similar observation was described during an outbreak in turkeys, where LPAI H5N1 isolates contained a deletion in the NA stalk region but an $\mathrm{H} 5 \mathrm{~N} 1$ isolate from a mallard encoded the fulllength NA gene [29].

It appears that NA stalk deletions linked to adaptation in the host chicken may be species-specific. For example, a deletion in the NA gene was only observed after passage of an H11N9 wild-bird LPAI isolate in chickens, whereas only a full-length NA sequence was detected after passage in ducks [21]. This species-specific event is also supported by a study that showed a requirement of 23 serial passages in quail to adapt an H9N2 isolate to be transmitted from chicken to chicken, whereas the transmission rate of this same isolate increased after ten serial passages in chickens [10]. Analysis of the viral sequences from that study revealed a number of mutations in the viral genes, but unique for the chicken-passaged isolate, the findings revealed a truncation of the NA stalk region.

The occurrence of genetic mutations that occur during influenza A virus passage is thought to be linked to the low fidelity of the viral polymerase [38]. However, the selection process of minority subpopulations from quasispecies that arise from the pool of viruses associated with the inoculum may be due to better fitness of the minor subpopulation in a new host, resulting in the emergence of a virus with a different phenotype. Thus, it is reasonable to postulate that mutations occur first, forming the quasispecies pool from which a distinct viral subpopulation emerges due to better fitness in the host, e.g., embryonated eggs, tissue culture, or an infected bird.

In the study reported here, changes in HA and NA genotype and resulting phenotype during passage of LPAI isolated from a wild-bird [A/Mute Swan/MI/451072/06 (H5N1)] or a chicken [A/Ck/TX/167280-4/02 (H5N3)] were investigated in chickens. The results support the hypothesis that, during viral infection of a host, a selection process occurs in which an existing minor population from a quasispecies arises from a virus pool. This argues against, but does not exclude, the idea that mutations occurring during passage in the parental virus result in the emergence of a virus with a different phenotype.

\section{Materials and methods}

Propagation of virus

Two H5 LPAI isolates, one from a chicken [A/Ck/TX/ 167280-4/02 (H5N3)] and one from a swan [A/Mute Swan/ MI/451072/06 (H5N1)], were kindly provided by Dr. David Suarez (South East Poultry Research Laboratory, Athens, GA, USA). A/Mute Swan/MI/451072/06 (H5N1) was isolated during monitoring programs in the United States [34], while the A/Ck/TX/167280-4/02 (H5N3) isolate was obtained from a chicken [20]. Both viruses were passaged two or three times before we performed a single propagation in 9-day-old embryonated chicken eggs (ECE) obtained from a specific-pathogen-free (SPF) leghorn chicken flock (Sunrise Farms, Catskill, NY, USA). The allantoic fluid from this parental stock was tested for hemagglutinating (HA) activity. HA-positive allantoic fluids were pooled, aliquoted, and stored at $-80^{\circ} \mathrm{C}$ until use. The $50 \%$ egg infectious dose $\left(\mathrm{EID}_{50}\right)$ for each isolate was determined by inoculating 10 -fold serial dilutions of allantoic fluid using 9-day-old embryonated SPF eggs, and the virus titer was calculated following as described previously [33].

\section{Infection experiments}

Three-week-old SPF leghorn chickens (Merial Select, Gainsville, GA, USA) were used for the infection experiments. All animal studies were conducted in BSL-2 $\mathrm{Ag}^{+}$ USDA-APHIS-approved animal facilities at the Poultry Diagnostic and Research Center at the University of Georgia. The animal experiments were approved by the Institutional Animal Care and Use Committee of the University of Georgia. The experiments were performed in isolators with a metal grid, with HEPA-filtered supply and exhaust air. Water and food were supplied ad libitum. Five three-week-old SPF chickens were infected via the choanal/intranasal route with a dose equivalent of $10^{6} \mathrm{EID}_{50}$. Twenty-four hours postinfection, five 22-day-old SPF birds were added as contacts. At day 2 (day 1 for contact birds), day 4 (day 3 for contact birds), day 7 (day 6 for contact birds), and day 9 (day 8 for contact birds) after infection, a tracheal-pharyngeal and a cloacal swab were taken from each bird and transferred to $2 \mathrm{ml}$ of viral transport medium (1x minimal essential medium, $7.5 \%$ sodium bicarbonate, $15 \mathrm{mM}$ HEPES, $1 \%$ fetal bovine serum, $4,000 \mathrm{U} / \mathrm{ml}$ penicillin, $400 \mu \mathrm{g} / \mathrm{ml}$ gentamycin, $8 \mu \mathrm{g} / \mathrm{ml}$ amphotericin $\mathrm{B}$, $4,000 \mu \mathrm{g} / \mathrm{ml}$ streptomycin, $1000 \mu \mathrm{g} / \mathrm{ml}$ kanamycin sulfate). The birds were bled before infection, and at day 21 pi via the brachial vein. At day 21 pi, the birds were euthanized according to a protocol approved by the Institutional Animal Care and Use Committee of the University of Georgia. 
Table 1 Oligonucleotides used for the analysis of HA and NA genes

\begin{tabular}{|c|c|c|c|c|c|c|}
\hline Name & Sequence & Gene $^{a}$ & Reaction $^{\mathrm{b}}$ & Orientation & Location $^{\mathrm{c}}$ & $\begin{array}{l}\text { GenBank } \\
\text { accession no }\end{array}$ \\
\hline UGAHA-F & AGCAAAAGCAGGGGTCYAWACTATSAAA & $\begin{array}{l}\text { HA-H5N1 } \\
\text { HA-H5N3 }\end{array}$ & RT-PCR,seq & Sense & $1-28$ & CY034679 \\
\hline UGAHA-R & AGTAGAAACAAGGGTGTTTTTAATTATAATCTG & $\begin{array}{l}\text { HA-H5N1 } \\
\text { HA-H5N3 }\end{array}$ & RT-PCR,seq & Antisense & $1735-1767$ & CY034679 \\
\hline MIHA-302F & TGGTCATACATCGTGGAAAAA & HA-H5N1 & seq & Sense & $302-322$ & CY034679 \\
\hline MIHA-620R & TGTTTGTTCAGCTGCATCATT & HA-H5N1 & seq & Antisense & $620-640$ & CY034679 \\
\hline MIHA-821F & GCTCCTGAATATGCGTACAAAA & HA-H5N1 & seq & Sense & $821-842$ & CY034679 \\
\hline MIHA-1135R & ATCCACTTCССТGCTCATTG & HA-H5N1 & seq & Antisense & $1135-1454$ & CY034679 \\
\hline MINA-425F & TCAAGGGGCCTTATTGAATG & NA-H5N1 & seq & Sense & $425-444$ & CY034680 \\
\hline MINA-898F & GAGACAACTGGCATGGTTCA & NA-H5N1 & seq & Sense & $898-917$ & CY034680 \\
\hline MINA-751R & CTGGCCATTACTTGGTCCAT & NA-H5N1 & seq & Antisense & $751-770$ & CY034680 \\
\hline MINA-1250R & AATCCAACCCCGTCAGTTCT & NA-H5N1 & seq & Antisense & $1250-1269$ & CY034680 \\
\hline TXHA-334F & GTGCTATCCAGGAGGCTTCA & HA-H5N3 & seq & Sense & $334-362$ & CY034683 \\
\hline TXHA-694R & TGACCGGGATTGACCTCTTA & HA-H5N3 & seq & Antisense & $694-713$ & CY034683 \\
\hline TXHA-863F & GCAATCATGAAAAGTGAACTGG & HA-H5N3 & seq & Sense & $863-884$ & CY034683 \\
\hline TXHA-1359F & TGGAAAATGAAAGAACTCTGGA & HA-H5N3 & seq & Sense & $1359-1380$ & CY034683 \\
\hline TXHA-1177R & TCCCATCAATTGCTTTCTGG & HA-H5N3 & seq & Antisense & $1177-1196$ & CY034683 \\
\hline TXNA-345F & GGGAGCACTGCTAGGGACTA & NA-H5N3 & seq & Sense & $345-364$ & CY034684 \\
\hline TXNA-688R & TCCAGTAAATCCTGTGATCTGC & NA-H5N3 & seq & Antisense & $688-709$ & CY034684 \\
\hline TXNA-873F & GGAAACAGGGTATGTTTGCAG & NA-H5N3 & seq & Sense & $873-893$ & CY034684 \\
\hline TXNA-1176R & AGGGCTGAAAACAGTCCTTG & NA-H5N3 & seq & Antisense & $1176-1195$ & CY034684 \\
\hline N1-FP & CCAAATCAAAAGATAATAACTATTGG & NA-H5N1 & RT-PCR & Sense & $27-52$ & CY034680 \\
\hline N1-RP & AAAGAAGGTCCTGCATTCCAAGTGAGAGC & NA-H5N1 & RT-PCR & Antisense & $391-419$ & CY034680 \\
\hline N1-INT & CAGACATACATCAACATAAGTAATACC & NA-H5N1 & RT-PCR & Sense & $210-236$ & CY034680 \\
\hline N1-Bridge & gcaatcaaagtgtcattacctgcagg & NA-H5N1 & RT-PCR & Sense & $166-185,245-250$ & CY034680 \\
\hline
\end{tabular}

a The viruses were A/Mute Swan/MI/451072/06 (H5N1) and A/Ck/TX/167280-4/02 (H5N3)

b The oligonucleotides were used for sequencing (seq) and/or for reverse transcription-polymerase chain reaction (RT-PCR)

c The location of the oligonucleotides is in accordance with the corresponding full-length sequence, as indicated by the GenBank accession number

The tracheal-pharyngeal and cloacal swab samples were centrifuged at $16,000 \mathrm{x} \mathrm{g}$, and $0.1 \mathrm{ml}$ of the supernatant was used for immediate inoculation of 9-day-old embryonated SPF eggs. The eggs were incubated for 4 days at $37^{\circ} \mathrm{C}$. The remaining swab samples were aliquoted and stored at $-80^{\circ} \mathrm{C}$. The eggs were candled daily, and embryo mortality observed within $24 \mathrm{~h}$ after inoculation was counted as nonspecific. The remaining eggs were investigated for the presence of influenza virus using the HA assay as described by OIE [26] after they were incubated for at least $4 \mathrm{~h}$ at $4^{\circ} \mathrm{C}$. Serum samples were investigated for the presence of hemagglutination-inhibiting (HI) antibodies by applying the HI test [26], using the homologous virus as the antigen. One-milliliter samples of each positive allantoic fluid sample from the same passage were pooled, and the virus pool was mixed by vortexing, aliquoted, and stored at $-80^{\circ} \mathrm{C}$. One sample was used for the determination of the $\mathrm{EID}_{50}$ following the method of Reed and Muench [33]. One sample from this pool was used for subsequent infection in the next passage level, again using $10^{6} \mathrm{EID}_{50}$ per bird.
Sequence analysis of the HA and NA genes

To analyze possible changes in both glycoproteins (HA and NA), the appropriate nucleotide sequences of the open reading frames were analyzed for each sample (swab and the appropriate allantoic fluid) that had tested positive by the HA test. To this end, the RNA from each positive sample was purified using an RNeasy purification kit (QIAGEN, Germantown, MD, USA) following the manufacturer's protocol. To amplify the appropriate cDNA fragments encompassing the open reading frames (ORFs) of the HA and NA proteins, reverse transcription-polymerase chain reaction (RT-PCR) was performed using a One-Step RT-PCR Kit (QIAGEN) as per the manufacturer's suggested protocol. The amplification of the HA ORF was performed using the primer pair UGAHA-F/ UGAHA-R (see Table 1). For amplification of N1 and N3 sequences, two previously published NA-specific primer pairs were used [12]. The RT-PCR fragments that were obtained were separated on a $1 \%$ agarose gel and eluted using a QIAquick Gel Extraction Kit (QIAGEN). The PCR 
fragments were sequenced directly using several oligonucleotides (see Table 1) to obtain two-fold coverage for each nucleotide sequence. Sequencing was performed using a BigDye Terminator v3.1 Cycle Sequencing Kit (Applied Biosystems, Foster City, CA, USA). The sequence reactions were purified using a Cleanseq magnetic bead system (Agencourt Bioscience Corporation, Beverly, MA, USA) and analyzed on an ABI 3130XL Genetic Analyzer (Applied Biosystems, Foster City, CA, USA) using an $80-\mathrm{cm}$ capillary array. The sequences obtained were analyzed using the Sequencher software (Genecodes Corporation, Ann Arbor, MI, USA). The location of the observed mutations in the HA protein was predicted using the Pymol program (The PyMOL Molecular Graphics System, Version 1.2, Schrödinger, LLC) based on the structure of an HA protein (protein data bank code 2IBX) that was published previously [39].

\section{Analysis of the deletion in the NA gene}

To investigate the deletion in the NA gene in A/Mute Swan/MI/451072/06 (H5N1) isolates, several reverse transcription-polymerase chain reaction (RT-PCR) experiments were performed. RNA from swab samples that resulted in virus isolation and RNA from the appropriate allantoic fluid samples were purified using a High Pure RNA Isolation Kit (Roche Diagnostics GmbH, Mannheim, Germany). The resulting RNA was used for single-step RT-PCR with Platinum Taq (Invitrogen, Carlsbad, CA, USA). For analysis of the NA truncation, three primer pairs were used. The first primer pair recognized sequences located upstream (N1-FP) and downstream (N1-RP) of the deleted nucleotide sequence (Fig. 3a), and PCR using these primers resulted in a cDNA fragment of $394 \mathrm{bp}$ for the fulllength N1 sequence and 334 bp for the truncated fragment. A third oligonucleotide, located within the deleted part of the N1 sequence (N1-INT), was used in combination with N1-RP, resulting in the amplification of an RT-PCR fragment only when the full-length N1 sequence (209 bp) was present. A fourth oligonucleotide (N1-bridge) was designed to amplify only the truncated version of the N1 nucleotide sequence in combination with N1-RP. The first 20 nucleotides of the oligonucleotide were located at the 5 'end, and the last six nucleotides were located immediately after the deleted sequence. The expected RT-PCR fragment size was $194 \mathrm{bp}$. Amplified RT-PCR fragments were gel-eluted as described above and cloned into the pCR2.1 plasmid using a TOPO TA Cloning Kit (Invitrogen). Plasmids containing an appropriate fragment were sequenced in both directions with M13 and M13R oligonucleotides using a BigDye Terminator v3.1 Cycle Sequencing Kit (Applied Biosystems). The products of the sequencing reactions were purified using Performa ${ }^{\circledR}$ DTR
Gel Filtration Cartridges (Edge Biosystems, Gaithersburg, MD, USA). Sequence analysis was performed using DNAStar Lasergene software (DNAStar Inc, Madison, WI, USA). The location of the primers used for this experiment is depicted (Fig. 4a), and the primer sequences are shown in Table 1.

\section{Serial passage of H5N1 in embryonated eggs}

The parental virus, A/Mute Swan/MI/451072/06 (H5N1), was serially passaged in embryonated chicken eggs. For each passage, five eggs were used. The initial passage was performed by infecting five nine-day-old embryonated eggs with $10^{5} \mathrm{EID}_{50} \mathrm{H} 5 \mathrm{~N} 1$ virus in a $100-\mu \mathrm{l}$ volume, and the eggs were incubated at $37^{\circ} \mathrm{C}$ for 4 days. Allantoic fluid was obtained and tested for HA activity. Allantoic fluids that tested positive were pooled and diluted 100-fold in phosphate-buffered saline, and $100 \mu \mathrm{l}$ was used for subsequent egg inoculation as described previously. Allantoic fluid from the fourth passage was taken individually from each egg and used for RT-PCR using the primers N1-FP/N1-RP and N1-bridge/N1-RP as described above.

\section{Results}

Passage studies in chickens

The six passage experiments (pas1-pas6) in chickens using the wild-bird isolate (A/Mute Swan/MI/451072/06 (H5N1), H5-WB, Fig. 1a, c) or the chicken isolate $(\mathrm{A} / \mathrm{Ck} / \mathrm{TX} /$ 167280-4/02 (H5N3), H5-Ck, Fig. 1b, d) were performed in parallel. The loss of birds infected with passage 2 (pas2) and pas3 H5-WB, and during the initial passage of $\mathrm{H} 5-\mathrm{Ck}$, was not likely related to influenza infection since no detectable macroscopic lesions specific for AIV infection were observed during necropsy. Virus was back-isolated from birds infected with $\mathrm{H} 5-\mathrm{Ck}$ (Fig. 1b) at day 2 and 4 pi during the passages, and the number of birds from which the virus was back-isolated varied between $1 / 5$ and $4 / 5$ of the infected birds. A similar pattern was observed in chickens infected with H5-WB (Fig. 1a), except that virus was back-isolated from 2 of the 5 infected birds at day 7 pi during pas1. Furthermore, the data were analyzed by ANOVA (http://www. danielsoper.com/statcalc/calc43.aspx). The mean events of virus isolation of each passage were compared between the two groups at each passage level. The number of backisolation events was only statistically different $(p<0.005)$ during the third and fourth passage, while during all other passages, the back-isolation rates were not statistically different $(\mathrm{p}>0.4)$. The statistical analysis indicates that the WB virus did not replicate better during the different passages, since in the first and second passages and the fifth 
a $\quad$ A/Mute Swan/ MI/ 451072/06 (H5N1)

No of chicken
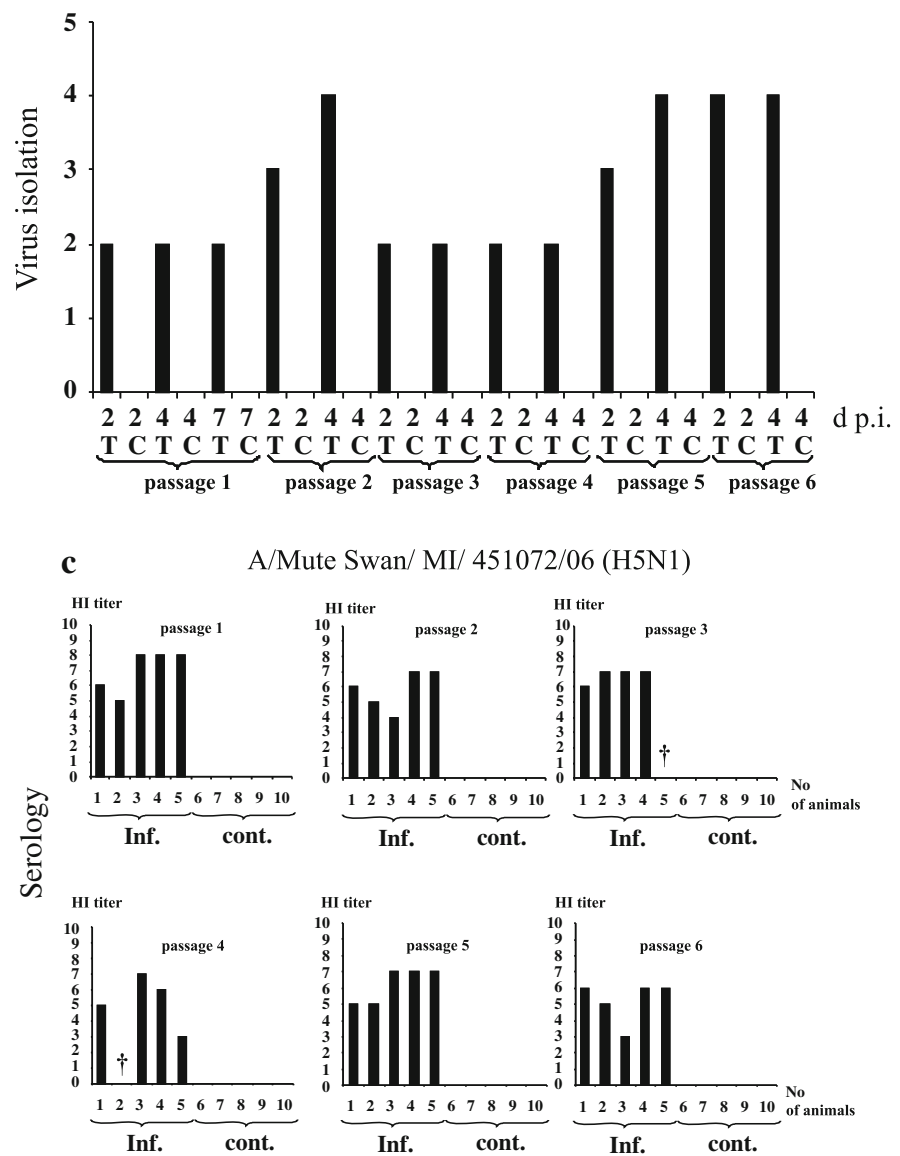

Fig. 1 Infection and replication of A/Mute Swan/ MI/ 451072/06 (H5N1) and A/Ck/TX/167280-4/02 (H5N3) during passage in chickens. Five 21-day-old chickens [1-5] were infected via the choanal /intranasal route with an LPAI wild-bird isolate [A/Mute Swan/ MI/ 451072/06 (H5N1)] or an LPAI isolate from a chicken (A/Ck/TX/167280-4/02 (H5N3)). Twenty-four hours after infection five 22-day-old hatchmates were added as contact birds [6-10]. Virus isolation was performed as described in Materials and methods from tracheal/oropharyngeal swabs

and sixth passages, no differences were observed. The results show that both viruses replicated in all infected chickens, as indicated also by seroconversion (Fig. 1c, d). The virus titers $\left(\mathrm{EID}_{50}\right)$ in the positive swab samples did not differ significantly between $\mathrm{H} 5-\mathrm{Ck}$ and $\mathrm{H} 5-\mathrm{WB}$ or between the different passage levels. The titers ranged between $10^{2}$ and $10^{3}$, and the EID $_{50}$ did not change over the course of the experiments.

Comparing HI titers of chickens infected with H5-WB (Fig. 1c) vs H5-Ck (Fig. 1d), there was a trend toward higher HI titers in chickens infected with H5-Ck. Virus was back-isolated from several birds in both groups of infected chickens at different frequencies, but no differences in the frequency of virus back-isolation was observed between the groups or between the different passages within the

\section{b $\quad \mathrm{A} / \mathrm{Ck} / \mathrm{TX} / 167280-4 / 02(\mathrm{H} 5 \mathrm{~N} 3)$}

No of chicken

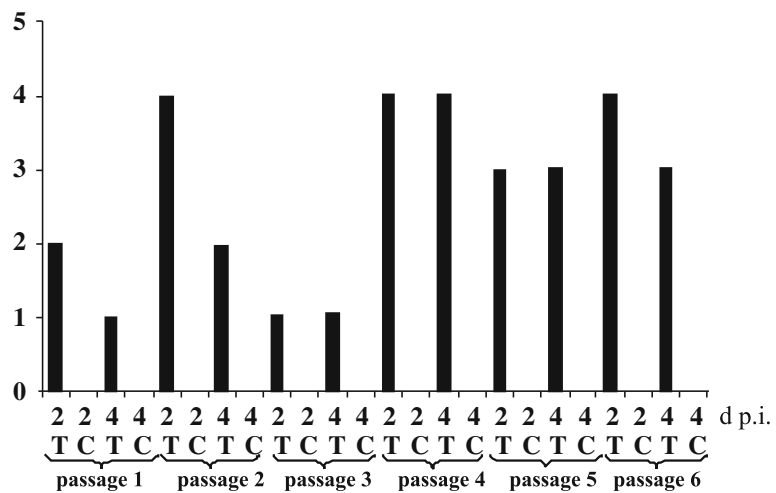

d

$\mathrm{A} / \mathrm{Ck} / \mathrm{TX} / 167280-4 / 02$ (H5N3)
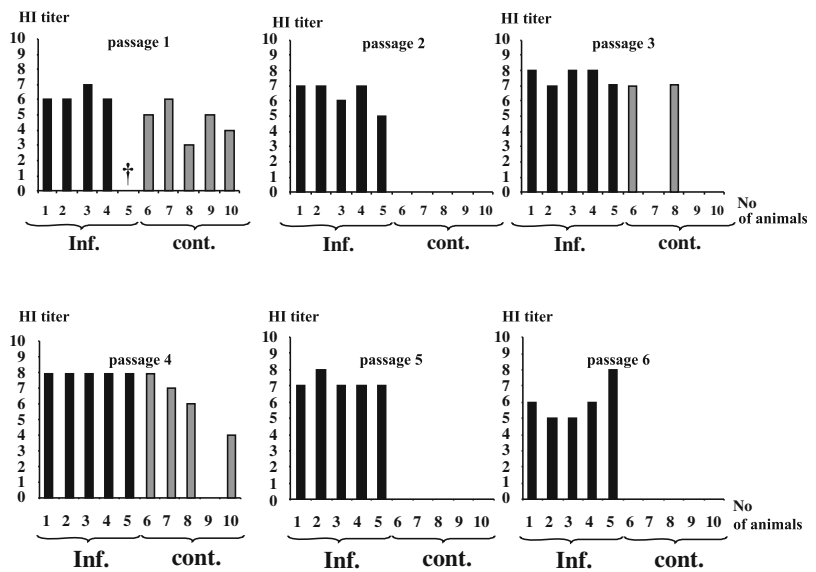

(T) and cloacal swabs (C). Virus was isolated only from infected birds (number of birds is represented by a bar) from passage 1 through passage 6. Serology was performed on serum samples taken from all birds at $21 \mathrm{~d}$ p.i. by $\mathrm{HI}$ test using the homologous antigen. The HI titer is shown as the reciprocal of the $\log 2$ value. Birds that died during the study are indicated by a cross. The HI titer of infected birds is shown by black bars, whereas the HI titer of the contact birds is shown by grey bars

groups. Interestingly, although seroconversion was evident following pas1, pas3, and pas4 in chickens infected with H5-Ck, no virus was back-isolated from contact birds. These results indicate that virus transmission occurred but was not consistent throughout serial passage of $\mathrm{H} 5-\mathrm{Ck}$, and it was not observed during the passage of H5-WB. In addition, shedding of virus was only detected in oropharyngeal swabs and never in cloacal swabs, indicating that both viruses replicated more efficiently in the respiratory tract.

Analysis of HA and NA gene sequences

To determine potential mutations associated with passage of the viruses in chickens, HA and NA gene sequences 
were determined. All allantoic fluids that tested positive for hemagglutination activity and the corresponding swab samples were examined. The majority of mutations that occurred during the H5-WB passages resulted in amino acid (aa) exchanges in both HA and NA early during passage (Table 2). The aa exchanges were analyzed based on the combinations of exchanges. All samples (allantoic fluid as well as swab sample) were included in the analysis. Specifically, the aa exchanges in the HA gene were Ile73Val, Asp99Asn, Thr341Lys, and Lys393Thr, which were observed during pas1 and became established in the viral population. The amino acid exchanges at position 172 (Ala to Thr) and at position 403 (Thr to Asn) were observed later during passage (pas5), and subsequent samples from later passages retained these changes, whereas one aa exchange at position 394 (Val to Ile) was only observed during pas 2 . It became evident that certain aa exchange combinations became established and were the basis for the next aa mutation, culminating in a combination (Ile73Val, Asp99Asn, Ala172Thr, Thr341Lys, Lys393Thr) that was present in the majority of the HA sequence after pas6. None of the observed exchanges resulted in the loss or addition of a potential glycosylation site (http://www.expasy.org). However, a mutation at position 341 (Thr to Lys) resulted in the replacement of a hydrophilic aa with a basic aa, potentially adding to the polybasic nature of the HA0 cleavage site.

The mutations in the NA protein of H5-WB were more substantial (Table 2). During pas1, a 20-aa deletion in the stalk region was observed. This deletion was always accompanied by an aa exchange at position 52 (Ser to Asn) and resulted in the loss of four potential N-glycosylation sites. This combination of mutations was observed in three of eight samples isolated during pas 1 and was present in the majority of the samples from subsequent passages. In addition, during pas2 (in 1 of 8 samples), pas4 (in 3 of 8 samples), and pas5 (in 7 of 16 samples), an amino acid was exchanged at position 49 (Cys to Tyr) but this exchange was not observed in any of the samples at pas6. In total, ten different aa exchanges were detected in $\mathrm{HA}$ and NA when including the deletion in the stalk region as a single event, and eight of these became established in the viral population.

Mutations in the HA and NA genes detected as amino acid exchanges were also observed during passage of $\mathrm{H} 5-\mathrm{Ck}$ but occurred at a lower frequency (Table 2). HA amino acid exchanges were observed during pas 1 in five combinations, and there was one single-aa exchange (Gln174Arg). In contrast to H5-WB, only a few mutations became established during the passages (Asn170Asp, Gln174Arg). Most were only observed after pas 1 (Gly155Asp, Lys177Glu, Asn260Asp), and these were observed in only a few samples. In addition, at pas6, the aa exchange Ser216Phe was detected in only one of six samples analyzed, but in combination with another two aa exchanges (Gln174Arg, Lys174Glu), which was observed once during pas1. In the NA gene, the only amino acid exchanges occurred at position 100, whereas during pas1 and pas2, the mutations Asp100Glu and Asp100Gly, respectively, were observed. After pas3 only Asp100Glu became established in the virus population, with one exception at pas4, where one sample showed the exchange Asp100Gly. It needs to be mentioned that the H5-Ck isolate already contained a deletion in the NA protein, indicating an already established adaptation to chickens during an unknown number of passages [25]. In total, eight amino acids were exchanged in the HA and NA genes during the course of H5-Ck passaging, but only four aa exchanges became established.

SNP sequence changes observed in chromatograms

To determine whether the observed amino acid exchanges were due to mutations occurring after infection or due to selection of an already existing virus subpopulation in the parental inoculum used to infect the chickens, chromatograms of the fully sequenced HA and NA genes of the stock viruses were analyzed (Fig. 2). For the HA gene of the H5-WB isolate (Fig. 2, upper panel), examination of the chromatogram analysis revealed that three of ten aa exchanges detected were probably already present in the parental inoculum due to the presence of minor peaks in the chromatograms [Ile73Val (a), Asp99Asn (b), Lys393Thr (c)], whereas the NA gene chromatograms did not show any minor peaks for any mutations observed. Interestingly, all three became established in the final viral population, as evidenced by the sequences of the sixth passage. In contrast, the HA chromatograms of H5-Ck HA (Fig. 2, lower panel) revealed that two of the eight mutations exhibited minor peaks [Gln174Arg (d), Lys177Glu (e)] relative to the parental inoculum before chicken passage, and for the NA gene, the Asp100Glu [Fig. 2, (f)] mutation was also already present in the chromatogram. In contrast to the H5-WB virus, only two aa exchanges (Gln174Arg, Asp100Glu) became established during the passage. In addition, there was an $\mathrm{A} / \mathrm{G}$ change at position 652 in the H5-Ck HA gene, which would result in a Thr218Ala substitution; however, this subpopulation was not selected during subsequent passage in chickens, and the NA gene had a synonymous nucleotide substitution at position 981 (A to $\mathrm{G}$ ).

Detailed macromolecular structure analysis of the aa exchanges in the HA gene (Pymol; http://www.pymol.org/) revealed that most of the aa substitutions occurred in the globular head region of the protein. For H5-WB, aa substitutions at positions 73,99 , and 172 were localized in the globular head, whereas aa substitutions at positions 393 and 395 were localized to the stalk region of the HA2 


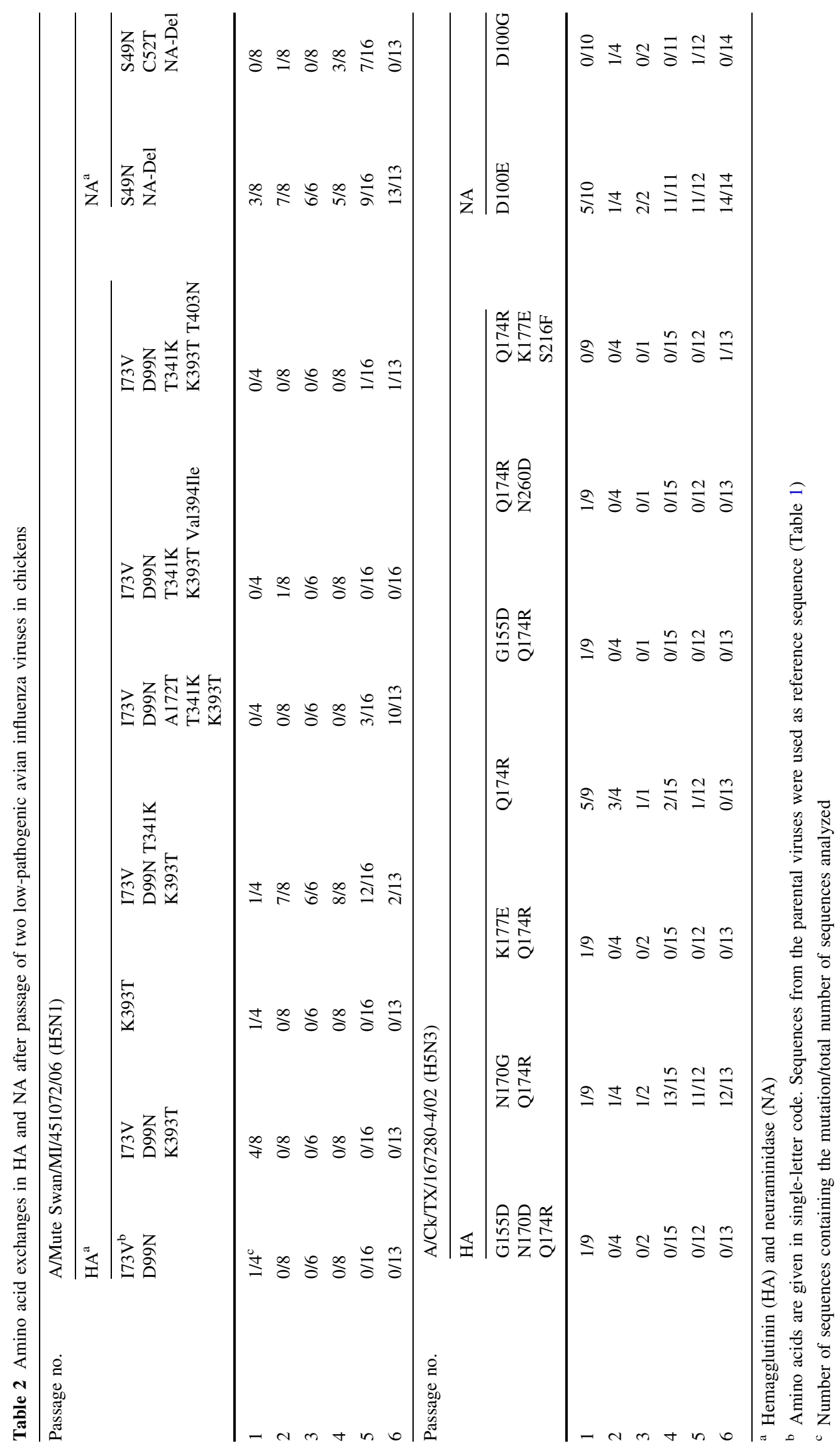


A/MuteSwan/MI/451072/06 (H5N1)

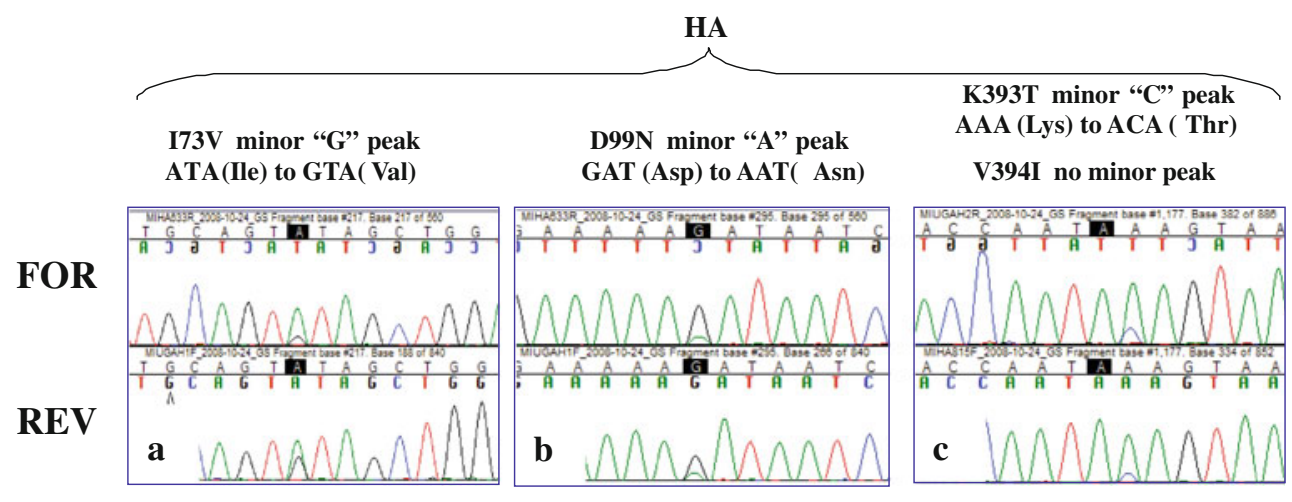

REV

$\mathrm{A} / \mathrm{Ck} / \mathrm{TX} / 167280$-4/02(H5N3)

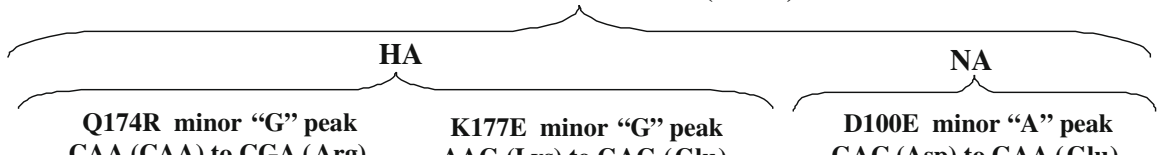

CAA (CAA) to CGA (Arg)

AAG (Lys) to GAG (Glu)

GAC (Asp) to GAA (Glu)
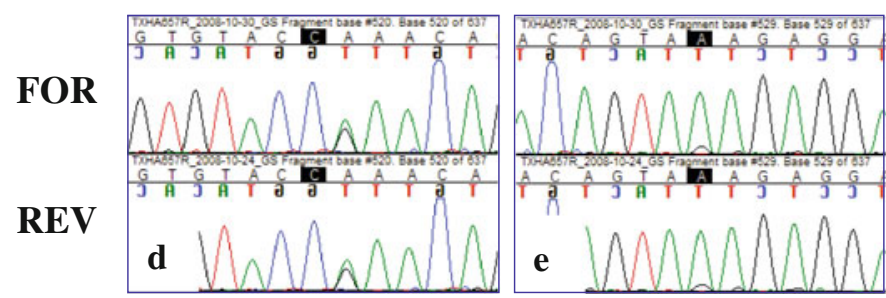

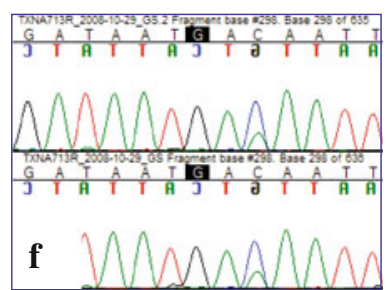

Fig. 2 Analysis of chromatograms of HA and NA genes sequences. Allantoic fluid from both viruses [A/Mute Swan/ MI/ 451072/06 (H5N1) or A/Ck/TX/167280-4/02 (H5N3)] was used for HA and NA gene sequence analysis. The amino acid exchanges observed during passage were analyzed for the presence of minor peaks present in the associated chromatograms of the forward (FOR) and reverse (REV) sequences. The observed minor sequencing peak representing the corresponding change in the codon is shown at the top of each chromatogram. The first nucleotide of the affected codon is indicated by a black box

expected RT-PCR fragment was generated using the internal primer (N1Frag-Int). Surprisingly, the primer pair that would generate the RT-PCR fragment representing the deleted form of the N1 gene (N1Frag-Del) gave a positive result using RNA from the stock parental virus. This result indicates that the parental virus population contained viruses that encoded either the deleted or the full-length form of the N1 gene. Using pas1 H5-WB samples, similar results were observed when N1Frag-Full, N1Frag-Int, and N1Frag-Del were detected. In contrast, RT-PCR using isolates from pas2 resulted only in the short form (N1Frag-Short) of the NA gene, i.e., $60 \mathrm{bp}$ shorter due to the deletion in the stalk region (Fig. 3a) when the flanking primer pair was used. Although the N1Frag-Int was still present, the amount of the amplified fragment was reduced. Also, the N1Frag-Del fragment was present, confirming the results of the first (parental stock virus) and second (pas1) RT-PCR analysis. The fragments from each lane were gel-eluted, cloned into pCR2.1, and sequenced to confirm the RT-PCR results. To rule out the possibility that the primer pair used for RT-PCR detection of the N1Frag-Del would be able to amplify a full-length N1 sequence, a PCR was performed using a plasmid containing 


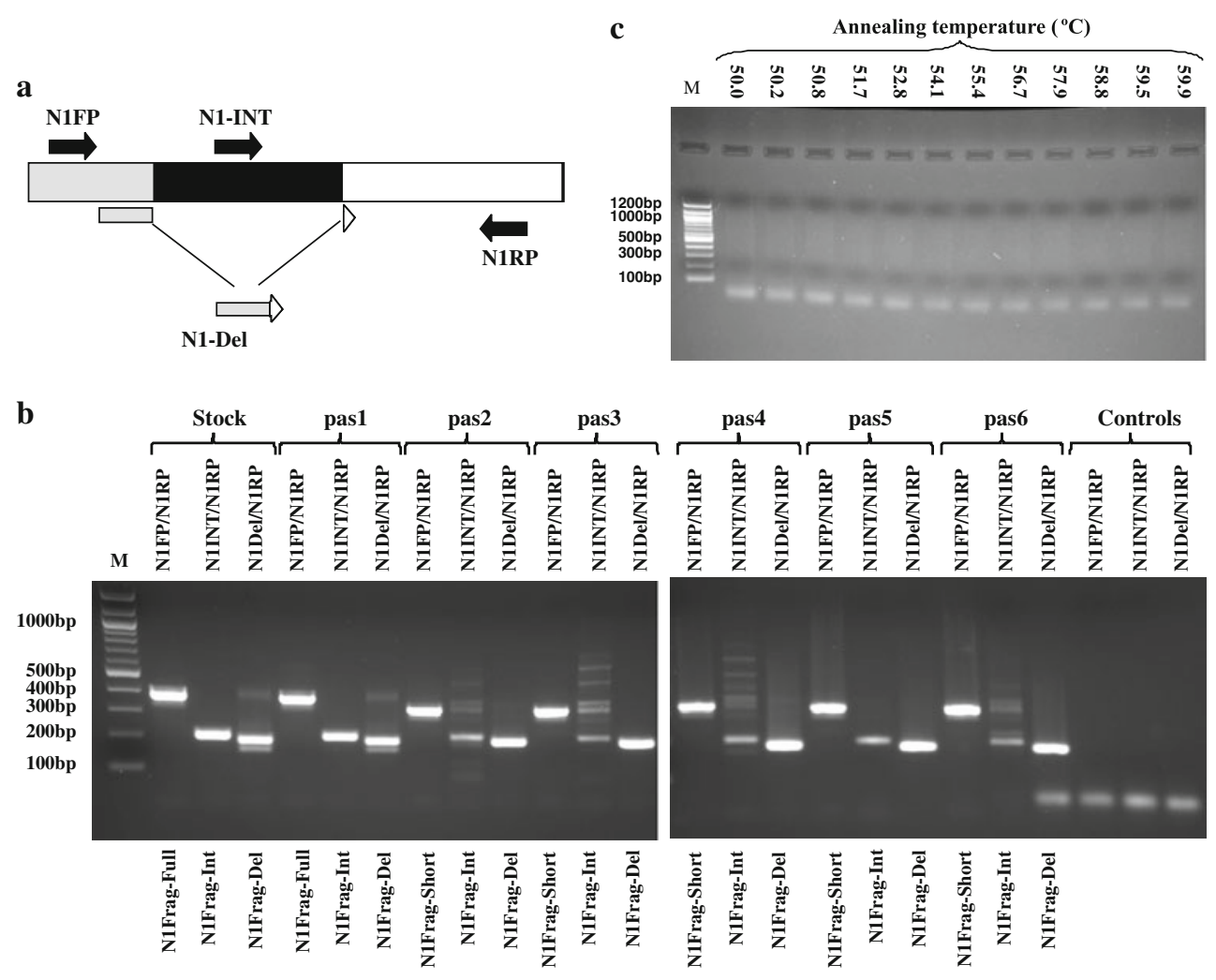

Fig. 3 Analysis of H5-WB NA gene quasispecies by RT-PCR. (a) The location of oligonucleotides is depicted in the schematic diagram. (b) Allantoic fluid samples from the parental virus and for each passage were analyzed by using three pairs of primers, N1FP/ N1RP, N1-INT/N1RP, N1-Del/N1RP. Allantoic fluid of an uninfected embryonated egg was used as negative control. Amplified fragments representing the full-length fragment (N1Frag-Full), the fragment that is shorter due to the 60-bp deletion (N1Frag-Short), the internal

N1Frag-Full. PCR performed using the same parameters as the RT-PCR assay except for the annealing temperature confirmed that the RT-PCR primer pair was unable to amplify a full-length $\mathrm{N} 1$ fragment under these conditions (Fig. 3c).

Further experiments were performed to determine if the form of NA with the 20-aa truncation could be selected during passage in embryonated eggs. To this end, four consecutive passages were performed in embryonated eggs. HA-positive samples from each passage were pooled and used for the subsequent passage to prevent artificial selection of viral subpopulations. At the fourth passage, allantoic fluid from each egg was used to determine if a switch from the majority (full-length N1) to the minority (truncated N1) had occurred (Fig. 4). The results from the RT-PCR show that only the 392-bp fragment was amplified, which indicates that during four passages in embryonated eggs, no change in the distribution of the N1 populations occurred. This suggests that the 20-aa NA stalk deletion in the pool of H5-WB quasispecies arises from a fragment (N1Frag-Int), and the fragment representing the deletion (N1-Del) were detected using the appropriate primer pairs. (c) PCR analysis using a temperature gradient in the annealing step was performed using the primer pair N1-Del/N1RP on a plasmid containing a DNA fragment representing the full-length sequence. The reaction products in $\mathrm{B}$ and $\mathrm{C}$ were analyzed on a $1.5 \%$ agarose gel along with a 100-bp ladder

minority subpopulation by an unknown selection mechanism to become dominant when passaged in chickens.

\section{Discussion}

Genetic changes during AIV passage in animals are not predicable. Infrequently, passage of LPAI isolates belonging to the $\mathrm{H} 5$ or $\mathrm{H} 7$ subtype results in the generation of HPAI, such as $\mathrm{H} 5$ and $\mathrm{H} 7$ strains that have been associated with the highly pathogenic phenotype. In the laboratory, HPAI viruses have been established by pre-selection of large plaques in cell culture $[3,4,22]$, reverse genetics and passage in naïve chickens [11], and brain-to-brain passage of LPAI in chickens [23]. It is known that the presence of a polybasic cleavage site between the HA1/HA2 subunits of the HA protein is a key contributor to the pathogenicity of AIV [11, 22]; however, the presence of a polybasic cleavage site in the HA alone does not produce the HPAI phenotype $[6,15,27$, $35,37]$. It is likely that the change in the viral phenotype 


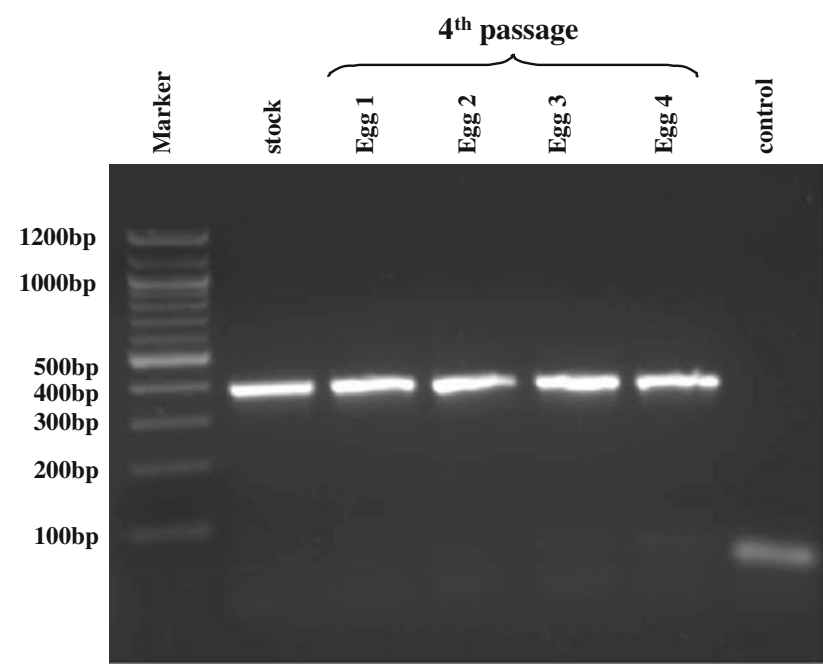

Fig. 4 The N1 deletion is not selected during passage in embryonated eggs. Allantoic fluid samples from the stock preparation of the parental virus (A/Mute Swan/ MI/ 451072/06 (H5N1)) and four eggs from the fourth passage of the virus were analyzed using primer pair N1FP/N1RP. Allantoic fluid from an uninfected embryonated egg was used as a negative control (control). The reaction products were analyzed on a $1.5 \%$ agarose gel. A 100-bp DNA ladder was used as a marker

from LPAI to HPAI is polygenic and associated with a gradual process and a spectrum of mutations.

Our study was designed to investigate changes in the viral genotype of the HA and NA genes after passage in chickens to determine if mutations that arose were linked to a change in phenotype. The results showed that after six passages, the H5-WB isolate was not transmitted from bird to bird, as evidenced by negative results for virus isolation and serology, although a number of mutations in the HA and NA genes, including the truncation in the N1 gene, were present, suggesting that the observed mutations were not sufficient to establish bird-to-bird transmission of the virus. H5-WB initially replicated in infected chickens at a rate similar to that shown previously for the same isolate [34]. In the study reported here, in later passages (pas2, pas5, pas6), it was observed that four of five infected chickens shed virus. Whether this was the result of an adaptation to the new host remains to be investigated in further experiments. In contrast, $\mathrm{H} 5-\mathrm{Ck}$ could be transmitted during the first, third, and fourth passage, as evidenced by seroconversion of the contact birds. Although isolation of virus from infected birds was expected, none was isolated from the contact birds, probably due to a very low rate of virus shedding, if any. Thus, the observed HA and NA gene sequence changes in H5-WB and H5-Ck were not sufficient during the six passages of each virus to facilitate consistent bird-to-bird transmission under these conditions. This means that additional passages will likely be necessary. It also needs to be taken into consideration that the conditions used in the isolator unit, which had a high air exchange rate, might not be optimal for supporting transmission from bird to bird. Thus, the husbandry, especially the airflow rate, might be an important factor for transmission. However, transmission of LPAI from chicken to chicken may vary, since replication of these viruses differs between virus isolates. For example, it has been shown that only 5 of 16 LPAI isolates from wild birds were able to infect chickens, as indicated by serological results [24]. In contrast, in a different study, one H11N9 isolate from a wild bird was shown to replicate in chickens and ducks and be efficiently transmitted to naïve hatchmates of both species [21]. However, similar to the results in this study, a recent study showed that an H9N2 isolate from a duck was not able to be transmitted from chicken to chicken, and only after 23 passages in quail using a lung homogenate for each subsequent infection was chicken-tochicken transmission observed [10]. These studies indicate that there is substantial variability between LPAI virus isolates with respect to their ability to infect chickens and be transmitted from chicken to chicken. Although the observed mutations were not sufficient to confer transmission between chickens, it is notable that the majority of the observed HA and NA mutations were detected by sequencing early after passage and remained relatively stable throughout passage, a feature that prompted us to question whether these amino acid exchanges had occurred during passage or were the result of selection of virus subpopulations that were already present. Studies have shown single-nucleotide polymorphisms (SNP) in LPAI isolates, in samples from nasopharyngeal or endotracheal aspirates from humans infected with the recent pandemic influenza A (H1N1), and in samples obtained from patients infected with seasonal H3N2, indicating the presence of heterogenous virus populations per se in the appropriate species [10, 31, 41]. Similarly, in this study, several SNPs occurred at HA and NA positions that were selected during passage in chickens, but some of the SNPs that were found to be present in the parental inoculum were not selected for during passage. These findings support the hypothesis that minor virus populations are selected from a quasispecies during passage, and together with mutations that may occur during passage, a fitter virus arises in the host. These findings are consistent with the concept of positive selection, in which viral subpopulations present in a virus population are selected during passage [3, 4].

An example of positive selection in the study reported here was the outgrowth of the NA stalk-deletion mutant from the subpopulation already present in the parental virus inoculum. That this occurred due to positive selection rather than mutation due to passage is supported by the findings that selection of the already present truncated version of the NA gene had occurred early, at pas2, and 
that the full-length NA sequence was still detectable in the samples after pas6. These findings are contrary to a recent and related study in which the preexistence of NA stalk deletions in the parental virus inoculum was not found using primers located outside of the deletion region [21], and it was concluded that there is no evidence for the preexistence of a deletion in the stalk region.

The results of this study show that an LPAI H5 isolate from a wild bird, i.e., a mute swan, has a higher mutation rate, characterized by more amino acid exchanges in the HA and NA genes during passage in chickens, than an LPAI H5 isolate from a chicken. These results suggest a greater degree of mutation may be needed in the wild-bird isolate to enable transmission in chickens. However, as reported, most of the observed amino acid exchanges were already present in the inoculum virus population before the infection passage experiments. This might be an indication that viruses encoding these amino acids were already present in low quantities in wild birds, but this is only an assumption, since virus isolates from wild birds will have at least one egg passage in embryonated chickens eggs. The results indicate a process of positive selection of minor virus populations within the quasispecies, which probably arise from a virus population due to better fitness in the chicken.

Acknowledgements This research was supported by the NIAID Center of Excellence for Influenza Research and Surveillance contract HHSN266200700006C and the Georgia Research Alliance.

\section{References}

1. Banks J, Speidel ES, Moore E, Plowright L, Piccirillo A, Capua I, Cordioli P, Fioretti A, Alexander DJ (2001) Changes in the hemagglutinin and the neuraminidase genes prior to the emergence of highly pathogenic H7N1 avian influenza viruses in Italy. Arch Virol 146:963-973

2. Bosch FX, Garten W, Klenk HD, Rott R (1981) Proteolytic cleavage of influenza virus hemagglutinins: primary structure of the connecting peptide between HA1 and HA2 determines proteolytic cleavability and pathogenicity of avian influenza viruses. Virology 113:725-735

3. Brugh M (1988) Highly pathogenic virus recovered from chickens infected with mildly pathogenic 1986 isolates of H5N2 avian influenza virus. Avian Dis 32:695-703

4. Brugh M, Perdue ML (1991) Emergence of highly pathogenic virus during selective chicken passage of the prototype mildly pathogenic chicken/Pennsylvania/83 (H5N2) influenza virus. Avian Dis 35:824-833

5. Chen H, Wen X, To KK, Wang P, Tse H, Chan JF, Tsoi HW, Fung KS, Tse CW, Lee RA, Chan KH, Yuen KY (2010) Quasispecies of the D225G substitution in the hemagglutinin of pandemic influenza $\mathrm{A}(\mathrm{H} 1 \mathrm{~N} 1) 2009$ virus from patients with severe disease in Hong Kong, China. J Infect Dis 201:1517-1521

6. Deshpande KL, Fried VA, Ando M, Webster RG (1987) Glycosylation affects cleavage of an H5N2 influenza virus hemagglutinin and regulates virulence. Proc Natl Acad Sci USA 84:36-40
7. Domingo E, Baranowski E, Ruiz-Jarabo CM, Martín-Hernández AM, Sáiz JC, Escarmís C (1998) Quasispecies structure and persistence of RNA viruses. Emerg Infect Dis 4:521-527

8. Domingo E, Holland JJ (1997) RNA virus mutations and fitness for survival. Annu Rev Microbiol 51:151-178

9. Fouchier RA, Munster V, Wallensten A, Bestebroer TM, Herfst S, Smith D, Rimmelzwaan GF, Olsen B, Osterhaus AD (2005) Characterization of a novel influenza A virus hemagglutinin subtype (H16) obtained from black-headed gulls. J Virol 79:2814-2822

10. Hossain MJ, Hickman D, Perez DR (2008) Evidence of expanded host range and mammalian-associated genetic changes in a duck H9N2 influenza virus following adaptation in quail and chickens. PLoS One 3:e3170

11. Horimoto T, Kawaoka Y (1994) Reverse genetics provides direct evidence for a correlation of hemagglutinin cleavability and virulence of an avian influenza A virus. J Virol 68:3120-3128

12. Hofmann E, Stech J, Guan JY, Webster RG, Perez DR (2001) Universal primer set for the full-length amplification of all influenza A viruses. Arch Virol 146:2275-2289

13. Holland JJ, Spindler K, Horodyski F, Grabau E, Nichol S, VandePol S (1982) Rapid evolution of RNA genomes. Science 215:1577-1585

14. Hulse DJ, Webster RG, Russell RJ, Perez DR (2004) Molecular determinants within the surface proteins involved in the pathogenicity of H5N1 influenza viruses in chickens. J Virol 78: 9954-9964

15. Kawaoka Y, Naeve CW, Webster RG (1984) Is virulence of H5N2 influenza viruses in chickens associated with loss of carbohydrate from the hemagglutinin? Virology 139:303-316

16. Klenk HD, Rott R, Orlich M (1977) Further studies on the activation of influenza virus by proteolytic cleavage of the haemagglutinin. J Gen Virol 36:151-161

17. Lamb RA (1989) Genes and proteins of the influenza virus. In: Krug RM, Fraenkel-Conrat H, Wagner RR (eds) The influenza viruses, 5th edn. Plenum Press, New York, pp 1-88

18. Lamb RA, Choppin PW, Chanock RM, Lai CJ (1980) Mapping of the two overlapping genes for polypeptides NS1 and NS2 on RNA segment 8 of influenza virus genome. Proc Natl Acad Sci USA 77:1857-1861

19. Lamb RA, Lai CJ (1980) Sequence of interrupted and uninterrupted mRNAs and cloned DNA coding for the two overlapping nonstructural proteins of influenza virus. Cell 21:475-485

20. Lee CW, Senne DA, Linares JA, Woolcock PR, Stallknecht DE, Spackman E, Swayne DE, Suarez DL (2004) Characterization of recent $\mathrm{H} 5$ subtype avian influenza viruses from US poultry. Avian Pathol 33:288-297

21. Li J, zu Dohna H, Anchell NL, Adams SC, Dao NT, Xing Z, Cardona CJ (2010) Adaptation and transmission of a duck-origin avian influenza virus in poultry species. Virus Res 147:40-46

22. Li SQ, Orlich M, Rott R (1990) Generation of seal influenza virus variants pathogenic for chickens, because of hemagglutinin cleavage site changes. J Virol 64:3297-3303

23. Löndt BZ, Banks J, Gardner R, Cox WJ, Brown IH (2007) Induced increase in virulence of low pathogenic avian influenza by serial intracerebral passage in chickens. Avian Dis 51:396-400

24. Morales AC Jr, Hilt DA, Williams SM, Pantin-Jackwood MJ, Suarez DL, Spackman E, Stallknecht DE, Jackwood MW (2010) Biologic characterization of H4, H6, and $\mathrm{H} 9$ type low pathogenicity avian influenza viruses from wild birds in chickens and turkeys. Avian Dis 53:52-562

25. Mundt E, Gay L, Jones L, Saavedra G, Tompkins SM, Tripp RA (2009) Replication and pathogenesis associated with H5N1, $\mathrm{H} 5 \mathrm{~N} 2$ and $\mathrm{H} 5 \mathrm{~N} 3$ low pathogenic avian influenza virus infection in chickens and ducks. Arch Virol 154:1241-1248

26. OIE (2009). http://www.oie.int/eng/normes/mmanual/2008/pdf/2. 03.04_AI.pdf (adopted version from May 2009) 
27. Ohuchi M, Orlich M, Ohuchi R, Simpson BE, Garten W, Klenk HD, Rott R (1989) Mutations at the cleavage site of the hemagglutinin after the pathogenicity of influenza virus A/chick/ Penn/83 (H5N2). Virology 168:274-280

28. O'Neill RE, Talon J, Palese P (1998) The influenza virus NEP (NS2 protein) mediates the nuclear export of viral ribonucleoproteins. EMBO J 17:288-296

29. Pei Y, Swinton J, Ojkic D, Sharif S (2009) Genetic characterization of two low pathogenic avian influenza virus H5N1 isolates from Ontario, Canada. Virus Genes 38:149-154

30. Perdue ML, Garcia M, Senne D, Fraire M (1997) Virulenceassociated sequence duplication at the hemagglutinin cleavage site of avian influenza viruses. Virus Res 49:173-186

31. Ramakrishnan MA, Tu ZJ, Singh S, Chockalingam AK, Gramer MR, Wang P, Goyal SM, Yang M, Halvorson DA, Sreevatsan S (2009) The feasibility of using high resolution genome sequencing of influenza A viruses to detect mixed infections and quasispecies. PLoS One 22:e7105

32. Richardson JC, Akkina RK (1991) NS2 protein of influenza virus is found in purified virus and phosphorylated in infected cells. Arch Virol 116:69-80

33. Reed LJ, Muench H (1938) A simple method of estimating fifty per cent endpoints. Am J Epidemiol 27:493-497

34. Spackman E, Swayne DE, Suarez DL, Senne DA, Pedersen JC, Killian ML, Pasick J, Handel K, Pillai SP et al (2007) Characterization of low-pathogenicity $\mathrm{H} 5 \mathrm{~N} 1$ avian influenza viruses from North America. J Virol 81:11612-11619
35. Suarez DL, Senne DA, Banks J, Brown IH, Essen SC, Lee CW, Manvell RJ, Mathieu-Benson C et al (2004) Recombination resulting in virulence shift in avian influenza outbreak Chile. Emerg Infect Dis 10:693-699

36. Stallknecht DE, Shane SM (1988) Host range of avian influenza virus in free-living birds. Vet Res Commun 12:125-141

37. Stech O, Veits J, Weber S, Deckers D, Schröer D, Vahlenkamp TW, Breithaupt A, Teifke J, Mettenleiter TC et al (2009) Acquisition of a polybasic hemagglutinin cleavage site by a low-pathogenic avian influenza virus is not sufficient for immediate transformation into a highly pathogenic strain. J Virol 83:5864-5868

38. Stech J, Xiong X, Scholtissek C, Webster RG (1999) Independence of evolutionary and mutational rates after transmission of avian influenza viruses to swine. J Virol 73:1878-1884

39. Yamada S, Suzuki Y, Suzuki T, Le MQ, Nidom CA, SakaiTagawa Y, Muramoto Y, Ito M, Kiso M et al (2006) Haemagglutinin mutations responsible for the binding of H5N1 influenza A viruses to human-type receptors. Nature 444:378-382

40. Webster RG, Bean WJ, Gorman OT, Chambers TM, Kawaoka Y (1992) Evolution and ecology of influenza A viruses. Microbiol Rev 56:152-179

41. Zhirnov OP, Vorobjeva IV, Saphonova OA, Poyarkov SV, Ovcharenko AV, Anhlan D, Malyshev NA (2009) Structural and evolutionary characteristics of HA, NA, NS and M genes of clinical influenza $\mathrm{A} / \mathrm{H} 3 \mathrm{~N} 2$ viruses passaged in human and canine cells. J Clin Virol 45:322-333 